\title{
Exclusive Top Threshold Matching at Lepton Colliders
}

Jürgen Reuter*

DESY, Hamburg, Germany

E-mail: juergen.reuter@desy. de

\section{Bijan Chokoufé Nejad}

DESY, Hamburg, Germany

E-mail: bijan.chokoufe@desy.de

\section{Andre Hoang}

University of Vienna, Vienna, Austria

E-mail: andre.hoang@univie.ac.at

\section{Wolfgang Kilian}

University of Siegen, Germany

E-mail: kilian@physik.uni-siegen.de

\section{Maximilian Stahlhofen}

Johannes-Gutenberg-University, Mainz, Germany

E-mail: mastahlh@uni-mainz.de

Thomas Teubner

University of Liverpool, United Kingdom

E-mail: thomas.teubneraliverpool.ac.uk

\section{Christian Weiss}

DESY, Hamburg, Germany

E-mail: christian.weissedesy.de

The threshold scan at future lepton colliders is the most precise known method to determine the top quark mass (well below $100 \mathrm{MeV}$ ), a fundamental parameter of the Standard Model that co-determines the stability properties of the electroweak vacuum. We present a new method to match the continuum next-to-leading order QCD corrections with the next-to-leading logarithmic resummation of the Coulomb singularities of the quasi-toponium bound state at threshold where fixed-order perturbation theory is invalid. This matching is performed at the level of the fully exclusive $\mathrm{WbWb}$ final state. It allows to study all kinds of differential distributions at or close to threshold. The top mass dependence of these distributions opens up new possibilities for the top mass determination that might be competitive with the inclusive threshold scan.

39th International Conference on High Energy Physics

4-11 July 2018

Seoul, Korea

${ }^{*}$ Speaker. 


\section{Introduction}

Future high-energy lepton colliders will measure the top quark properties like its mass, width and couplings with an unprecedented accuracy and use the top quark as a means to search for new physics beyond the Standard Model. Here, we discuss the matching of fixed-order QCD next-to-leading order (NLO-QCD calculations for exclusive $e^{+} e^{-} \rightarrow W^{+} b W^{-} \bar{b}$ final states in the continuum, based on [1], with a resummed calculation in the threshold region, where fixed-order perturbation theory in the strong coupling $\alpha_{s}$ is not a good approximation anymore, but the top velocity $v$ is an additional expansion parameter and Coulomb-singular terms $\sim\left(\alpha_{s} / v\right)^{n}$ and (ideally also) large logarithms $\sim\left(\alpha_{s} \log v\right)^{n}$ have to be resummed. In Sec. 2, after reviewing our calculational framework and the details of the continuum calculation for completeness, we discuss, based on [2], a previously known non-relativistic QCD (NRQCD) effective field theory setup to compute a form factor accounting for the resummation of the threshold-singular terms at NLL accuracy, implemented it in the fixed-order calculation and matched the result to the QCD-NLO cross section in the transition region between threshold and continuum. We thus obtained a fully-differential cross section, which gives reliable predictions for all center-of-mass energies. Depending on how inclusive the process is, we achieve LL + QCD-NLO (for very exclusive processes) or NLL + QCD-NLO precision (for inclusive processes) in the threshold region. Finally, we conclude in Sec. 3.

\section{QCD-NLO (fixed-order) \& Threshold Matching}

In the continuum, i.e. away from the threshold, QCD corrections are properly described by fixed-order relativistic QCD-NLO perturbation theory for the off-shell top pair production. For that purpose, we study either the process $e^{+} e^{-} \rightarrow W^{+} b W^{-} \bar{b}$ or $e^{+} e^{-} \rightarrow \ell^{+} e^{-} \bar{v}_{e} \mu^{+} v_{\mu} b \bar{b}$ including leptonic $W$ decays. Within the full four- or six-particle final state, there are double-resonant diagrams included (involving a top and an anti-top propagator), single-resonant diagrams and non-resonant irreducible background processes. To calculate total and fully differential QCD-NLO corrections for the top production processes, we take the WHIZARD framework for (QCD-)NLO processes. WHIZARD [3] is a multi-purpose event generator with its own matrix-element generator for treelevel amplitudes, $O^{\prime}$ Mega [4,5] with support for a plethora of models like e.g. supersymmetry [6]. Users can use external models by the interface to FeynRules [7]. WHIZARD uses the color-flow formalism [8], and it comes with its own parton shower implementation [9]. QCD-NLO applications within WHIZARD started with a hard-coded implementation for the production of $b$ jets at LHC [10, 11], while matching between resummed terms and fixed-order calculations have been tackled by combining fixed-order electroweak corrections to chargino production at the ILC with an all-order QED initial-state structure function $[12,13]$. WHIZARD is also able to do automatic POWHEG matching for $e^{+} e^{-}$processes [14].

WHIZARD uses FKS subtraction [15] and generates the automatically generates the phase space for all singular emission regions. Virtual matrix elements, color-correlated and spin-correlated matrix elements for the collinear and soft splittings are taken from the one-loop provider (OLP) program OpenLoops [16]. The complex mass scheme is used, leading to a complex weak mixing angles. The input values are as follows: $m_{W}=80.385 \mathrm{GeV}, m_{Z}=91.1876 \mathrm{GeV}, m_{t}=173.2$ 
$\mathrm{GeV}, m_{H}=125 \mathrm{GeV}$. We use massive $b$-quarks of mass $m_{b}=4.2 \mathrm{GeV}$. Widths need to be calculated at the same order and in the same scheme than the scattering process in order to guarantee properly normalized branching ratios: $\Gamma_{Z}^{\mathrm{LO}}=2.4409 \mathrm{GeV}, \Gamma_{Z}^{\mathrm{NLO}}=2.5060 \mathrm{GeV}, \Gamma_{W}^{\mathrm{LO}}=2.0454$ $\mathrm{GeV}, \Gamma_{W}^{\mathrm{NLO}}=2.0978 \mathrm{GeV}, \Gamma_{t \rightarrow W b}^{\mathrm{LO}}=1.4986 \mathrm{GeV}, \Gamma_{t \rightarrow W b}^{\mathrm{LO}}=1.3681 \mathrm{GeV}$. As the matrix elements for the full off-shell processes contain narrow resonances, particularly the $H \rightarrow b b$ resonance, we use a resonance-aware version of the FKS subtraction formalism to make sure that cancellations between real emissions and subtraction terms do cancel though the real emission could shift the kinematics on or off the resonance compared to Born kinematics. This resonance-aware treatment is automatically done in WHIZARD. As we are using massive $b$-quarks, no cuts are necessary for the process $e^{+} e^{-} \rightarrow W^{+} W^{-} b \bar{b}$. The integrations for the full QCD-NLO are very stable. We did two independent own integrations with the serial and the non-blocking MPI-parallelizable version [17] of VAMP [18] inside WHIZARD.

For the QCD-NLO corrections, we take the top mass as renormalization scale. The scale variations for the process $e^{+} e^{-} \rightarrow W^{+} b W^{-} \bar{b}$ is very small, at the level of two per cent. After one has replaced the top width in the matrix elements by a running top width $\Gamma_{t}\left(\mu_{R}\right)$, the scale variations for the on-shell process $e^{+} e^{-} \rightarrow t \bar{t}$ behave the same way as for the off-shell process. The WHIZARD infrastructure immediately enables QCD-NLO calculations/simulations for polarized beams, to include QED initial-state photon radiation as well as collider-specific beamspectra.

A kinematic fit to the shape of the rising of the cross section at the top threshold is believed to be the most precise method to measure the top quark mass with an ultimate precision of 30-80 $\mathrm{MeV}$. For this the systematic uncertainties of the experimental measurement - especially the details of the beam spectrum - as well as the theoretical uncertainties have to be well under control. As shown above, close to the kinematical threshold for the on-shell production of a $t \bar{t}$ pair, fixed-order
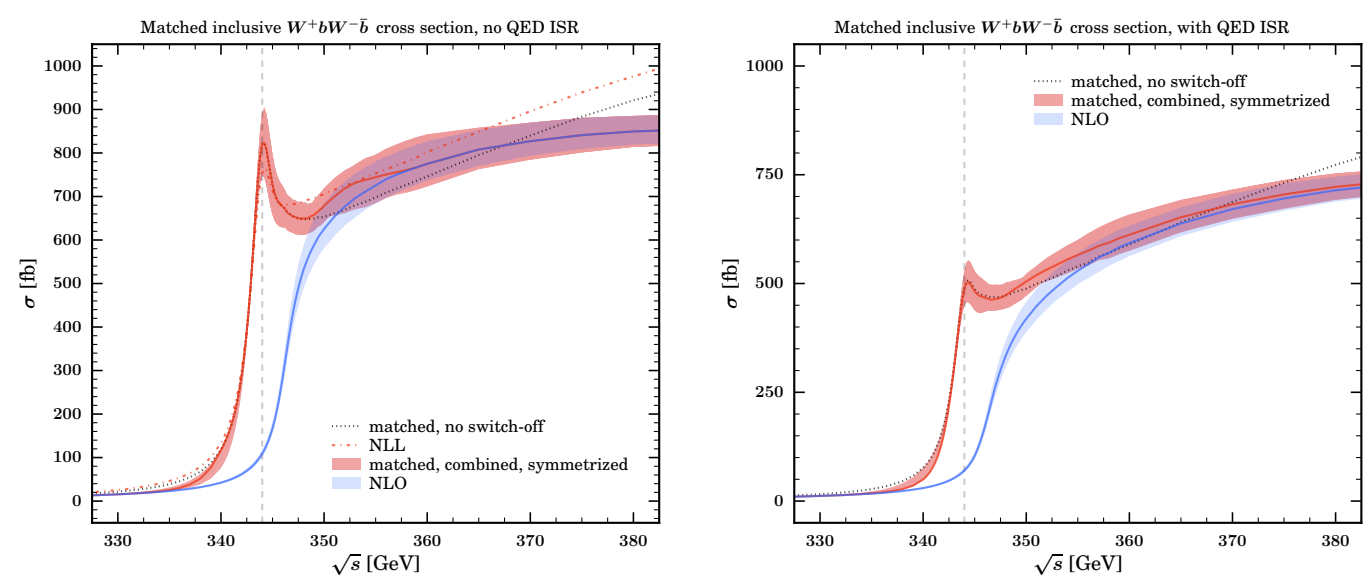

Figure 1: Matched NRQCD-NLL + QCD-NLO calculation without (left) and with (right) QED ISR. The dashed vertical line is the value of twice $M^{1 \mathrm{~S}}$. Blue is the fixed QCD-NLO calculation, red is the fully matched calculation. The matched calculation has a full envelope over (symmetrized) scale uncertainties as well as variations over switch-off functions.

perturbation theory is not a good approximation. Very close to threshold, the effective field theory of (v/p)NRQCD separates the hard scale $m_{t}$, the soft scale given by the top momentum of the nonrelativistic top quark with velocity $v, m_{t} v$ and the ultrasoft scale, given by the kinetic energy of the 
top quark, $m_{t} v^{2}$ and allows to resum large logarithms of $v$ with $\alpha_{s} \sim v \sim 0.1$ close to threshold. "Fixed-order" calculations resumming only Coulomb singularities, but no velocity logarithms, for the totally inclusive $t \bar{t}$ production have been carried out in NRQCD to NNNLO [19]. The large velocity logarithms have been resummed to next-to-next-leading logarithmic (NNLL) [22] order (cf. also [20, 21] for predictions not containing the full set of NNLL ultrasoft logarithms). These NRQCD calculations, based on the optical theorem, hold only for the total inclusive cross section in a narrow window around the $t \bar{t}$ threshold. Here, we combine and match the NLL NRQCDresummed process close to the top threshold with the fixed-order (relativistic) QCD-NLO process in the continuum. By a carefully performed matching procedure, our approach smoothly interpolates between threshold region and continuum, and allows to study all kinds of differential distributions.

The matching is embedded into the WHIZARD-OpenLoops QCD-NLO fixed-order framework discussed above. The NLL resummed NRQCD contributions are included in terms of (S-/Pwave) form factors to the (vector/axial vector) $\gamma / Z-t-\bar{t}$ vertex. These form factors are obtained from the numerical solution of Schrödinger-type equations for the NLL Green functions computed by the Toppik [23, 24, 25] code, which is included in WHIZARD, for technical details cf. [2]. In order to avoid double-counting between the fixed-order QCD-NLO part and the resummed NLLNRQCD part, one has to expand the form factors to first order in $\alpha_{s}$ and subtract those pieces. As the NRQCD resummed calculations are only available for the top-vector and axial-vector currents, this removal of double-counting has to be done in a factorized approach within a double-pole approximation. In order to maintain gauge-invariance of the factorized amplitudes, an on-shell projection of the exclusive final states to the top mass shell is performed, for details cf. [2]. The implementation inside WHIZARD has been validated with analytical calculations for different in-
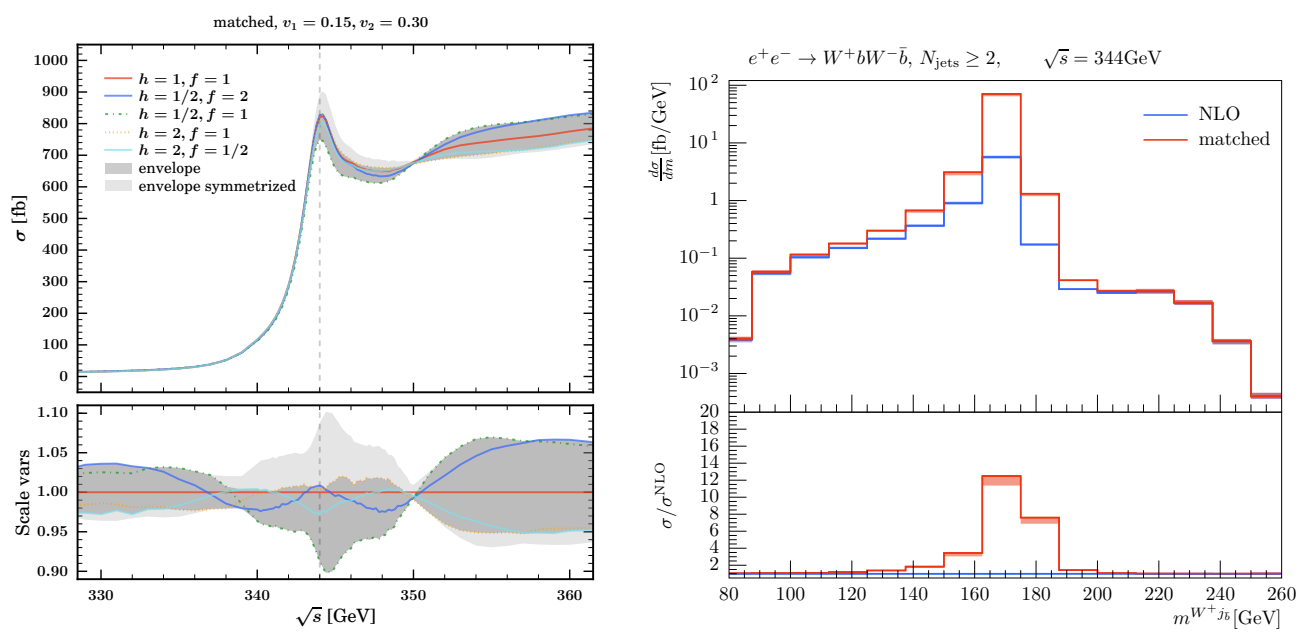

Figure 2: Left panel: Matched NRQCD-NLL + QCD-NLO total cross section as on the left of Fig. 1, but for a single choice of switch off-function. $h$ and $f$ are renormalization scale parameters as defined in $[2,22]$. The grey bands display the corresponding scale variations with and without symmetrization. Right panel: $W b$ invariant mass distribution at threshold $(\sqrt{s}=344 \mathrm{GeV})$ as obtained with WHI ZARD. The red line represents the full NRQCD-NLL + QCD-NLO matched, and the blue line the pure QCD-NLO result. The associated bands are generated by the same scale variations as in the left panel, here without symmetrization.

variant mass cuts on the reconstructed top quarks from Ref. [26]. 
For larger top velocity $(v \gtrsim 0.4)$ only the relativistic QCD-NLO result is valid. We define a switch-off function that smoothly interpolates between the two regions. The possibility to vary this arbitrary function and its parameters adds another theory uncertainty to the different scale variations [2]. The results of our matching procedure are displayed in Fig. 1. These plots show the total inclusive cross section for the process $e^{+} e^{-} \rightarrow W^{+} b W^{-} \bar{b}$, in the left panel without and in the right panel with QED initial-state radiation (ISR). The dashed vertical line gives the value for $2 M^{1 \mathrm{~S}}$. The $1 \mathrm{~S}$ mass $M^{1 \mathrm{~S}}$ is defined as half of the perturbative mass of a would-be $1 \mathrm{~S}$ toponium state and represents a renormalon free short-distance mass, which we treat as an input parameter in WHIZARD. The blue line shows the QCD-NLO cross section including scale variations in the blue shaded areas. The red curve shows the NRQCD-NLL + QCD-NLO result, while the shaded band contains all (symmetrized) scale variations of the hard, soft and ultrasoft factorization/renormalization scales according to [22] as well as variations of the switch-off function to a reasonable extent [2]. The dotted black line shows the matched results without applying a switch-off function to the factorized NRQCD terms which deviates above threshold from the relativistic QCD-NLO result. In Fig. 2, left panel, we see the matched result in the threshold region for a single choice of switchoff parameters, but scale variations over the full two-dimensional renormalization parameter range defined in [22]. This shows that the scale variation bands for the resummed NLL result in the threshold region are highly asymmetric with respect to the central value which motivates to apply a symmetrization of the error bands around the central value. This symmetrization is also shown in Fig. 1. In the right panel of Fig. 2 we show as an example for a differential distribution the invariant mass of the $W-b$ jet system. Blue is the fixed-order QCD-NLO distribution, while red is the fully matched distribution including scale variations, here un-symmetrized. The ratio plot in the bottom does not show a K factor, but the ratio of the matched result to the QCD-NLO fixed order result. It shows an enhancement in the top mass peak due to threshold resummation by a factor of 10-12.

\section{Conclusions}

In order to be able to study experimental event selections as well as differential distributions, we presented a matched threshold calculation that smoothly interpolates the threshold region described by non-relativistic QCD to the relativistic QCD-NLO calculation. It constitutes the highest precision available at the level of the completely exclusive final state. Any of the presented differential distributions depending on the top mass may serve as a different means to determine the top mass. We were not accomplishing this task here, but rather showed a framework as a proof-ofprinciple of the matching procedure between threshold and continuum. For the proper matching to the continuum the fixed-order QCD-NLO calculations for top-quark pair production including top and (leptonic) $W$ decays have been done. All of this has been done in the QCD-NLO framework of the WHIZARD event generator which allows to include all important physics of a lepton collider like polarization, QED ISR radiation and non-trivial beam spectra.

\section{References}

[1] B. Chokoufé Nejad, W. Kilian, J. M. Lindert, S. Pozzorini, J. Reuter and C. Weiss, JHEP 1612, 075 (2016) [arXiv:1609.03390 [hep-ph]]. 
[2] F. Bach, B. C. Nejad, A. Hoang, W. Kilian, J. Reuter, M. Stahlhofen, T. Teubner and C. Weiss, JHEP 1803, 184 (2018) [arXiv:1712.02220 [hep-ph]].

[3] W. Kilian, T. Ohl and J. Reuter, Eur. Phys. J. C 71, 1742 (2011) [arXiv:0708.4233 [hep-ph]].

[4] M. Moretti, T. Ohl and J. Reuter, hep-ph/0102195.

[5] B. Chokoufe Nejad, T. Ohl and J. Reuter, Comput. Phys. Commun. 196, 58 (2015) [arXiv:1411.3834 [physics.comp-ph]].

[6] T. Ohl and J. Reuter, Eur. Phys. J. C 30, 525 (2003) [hep-th/0212224].

[7] N. D. Christensen, C. Duhr, B. Fuks, J. Reuter and C. Speckner, Eur. Phys. J. C 72, 1990 (2012) [arXiv:1010.3251 [hep-ph]].

[8] W. Kilian, T. Ohl, J. Reuter and C. Speckner, JHEP 1210, 022 (2012) [arXiv:1206.3700 [hep-ph]].

[9] W. Kilian, J. Reuter, S. Schmidt and D. Wiesler, JHEP 1204, 013 (2012) [arXiv:1112.1039 [hep-ph]].

[10] T. Binoth, N. Greiner, A. Guffanti, J. Reuter, J.-P. Guillet and T. Reiter, Phys. Lett. B 685, 293 (2010) [arXiv:0910.4379 [hep-ph]].

[11] N. Greiner, A. Guffanti, T. Reiter and J. Reuter, Phys. Rev. Lett. 107, 102002 (2011) [arXiv:1105.3624 [hep-ph]].

[12] W. Kilian, J. Reuter and T. Robens, Eur. Phys. J. C 48, 389 (2006) [hep-ph/0607127].

[13] T. Robens, J. Kalinowski, K. Rolbiecki, W. Kilian and J. Reuter, Acta Phys. Polon. B 39, 1705 (2008) [arXiv:0803.4161 [hep-ph]].

[14] J. Reuter, B. Chokoufe, A. Hoang, W. Kilian, M. Stahlhofen, T. Teubner and C. Weiss, J. Phys. Conf. Ser. 762, no. 1, 012059 (2016) [arXiv:1602.06270 [hep-ph]].

[15] S. Frixione, Z. Kunszt and A. Signer, Nucl. Phys. B 467, 399 (1996) [hep-ph/9512328].

[16] F. Cascioli, P. Maierhöfer and S. Pozzorini, Phys. Rev. Lett. 108, 111601 (2012) [arXiv:1111.5206 [hep-ph]]; F. Buccioni, S. Pozzorini and M. Zoller, Eur. Phys. J. C 78, no. 1, 70 (2018) [arXiv:1710.11452 [hep-ph]].

[17] S. Braß, W. Kilian, J. Reuter, DESY 18-198, SI-HEP-2018-32, in preparation

[18] T. Ohl, Comput. Phys. Commun. 120, 13 (1999) [hep-ph/9806432].

[19] M. Beneke, Y. Kiyo, P. Marquard, A. Penin, J. Piclum and M. Steinhauser, Phys. Rev. Lett. 115 , no. 19, 192001 (2015) [arXiv:1506.06864 [hep-ph]].

[20] A. H. Hoang, A. V. Manohar, I. W. Stewart and T. Teubner, Phys. Rev. D 65, 014014 (2002) [hep-ph/0107144].

[21] A. Pineda and A. Signer, Nucl. Phys. B 762, 67 (2007) [hep-ph/0607239].

[22] A. H. Hoang and M. Stahlhofen, JHEP 1405, 121 (2014) [arXiv:1309.6323 [hep-ph]].

[23] M. Jezabek, J. H. Kuhn and T. Teubner, Z. Phys. C 56, 653 (1992).

[24] R. Harlander, M. Jezabek, J. H. Kuhn and T. Teubner, Phys. Lett. B 346, 137 (1995) [hep-ph/9411395].

[25] A. H. Hoang and T. Teubner, Phys. Rev. D 60, 114027 (1999) [hep-ph/9904468].

[26] A. H. Hoang, C. J. Reisser and P. Ruiz-Femenia, Phys. Rev. D 82, 014005 (2010) [arXiv:1002.3223 [hep-ph]]. 